\title{
What Do Reciprocals Mean?
}

\author{
Mary Dalrymple, Xerox PARC \\ Makoto Kanazawa, Stanford University \\ Sam Mchombo, University of California-Berkeley \\ Stanley Peters, Stanford University
}

\begin{abstract}
Research on reciprocals has uncovered a variety of semantic contributions that the reciprocal can make, creating problems for proposals that the reciprocal unambiguously means something weak (e.g., Langendoen 1978). However, there is no real evidence that reciprocals are ambiguous, despite previous claims to the contrary (e.g., Fiengo and Lasnik 1973). First, we classify the apparently heterogeneous list of meanings proposed in previous research into a natural taxonomy, showing how they arise from a small stock of logical operations and predicates. Second, we exhibit a partial ordering of the various reciprocal meanings according to logical strength, which we make crucial use of in determining what reciprocals mean in each specific context where they appear. Third, we hypothesize that a reciprocal statement expresses the strongest candidate meaning that is consistent with known properties of the relation expressed by the scope of the reciprocal. This hypothesis is supported by analysis of a large collection of examples we have gathered from various corpora.
\end{abstract}

\section{The reciprocal as a quantifier}

Starting with Dougherty (1974) and Fiengo and Lasnik (1973), a significant modern literature has grown up on the subject of what reciprocals mean. Langendoen (1978) made a valuable systematization of the discussion and endeavored to draw some firm conclusions. Important contributions have been made subsequently by, among others, Higginbotham (1980), Kański (1987), Roberts (1987), Lønning (1989), Moltmann (1992), and Schwarzschild (1992).

As we undertake a new synthesis of the expanded literature on the subject and draw our own conclusions, we want to begin by pointing out something that, though implicit in the literature we survey, has never been made explicit:

- Reciprocal expressions such as English each other and one another, Chicheŵa -an, Japanese (o)tagai, etc., are quantifiers.

We do not mean that reciprocals somehow give rise to two quantifiers - perhaps two universal quantifiers, or one universal and one existential quantifier. We mean rather that:

- Reciprocals express a single polyadic quantifier that binds two variables in its scope, both variables ranging over one set, the restricted domain of the quantification.

Thus a sentence like

(C) 1994 by Mary Dalrymple, Makoto Kanzzawa, Sam Mchombo and Stanley Peters Mandy Harvey and Lynn Santelmann (eds.), SALT IV 61-78, Ithaca, N.Y.: Comell University. 
(1) Tom, Dick and Harry saw each other expresses a proposition that might be symbolized

(2) $\operatorname{RECIP}^{\text {\{Tom, Dick, Harry }\}} x, y$ saw $(x, y)$

using the quantifier symbol RECIP, with its restricted domain $\{$ Tom, Dick, Harry $\}$ superscripted and the two variables it binds $x, y$ suffixed, all preceding the quantifier's scope.

In terms of standard generalized quantifier theory, RECIP is a quantifier of type (1,2); its meaning is a relation between sets $A$ of members of the universe of discourse and two-place relations $R$ on the universe of discourse (van Benthem, 1989). A formula

$$
\mathrm{RECIP}^{A} u, v \phi
$$

is true iff the relation RECIP holds between the set $A$ and the binary relation of which $[\lambda u, v . \phi]$ is the characteristic function. Like all generalized quantifiers of natural languages, RECIP is required to be conservative, in the sense that

$$
\operatorname{RECIP}(A, R) \text { iff } \operatorname{RECIP}(A, R \cap(A \times A))
$$

holds for all $A$ and $R$.

Our purpose will be to propose a definition for RECIP, illustrating our claims with sentences taken from corpora we have examined. Section 3 examines previous proposals for reciprocal meaning. Most of these proposed meanings can be stated in terms of a set of simple relations. This allows for a specification of entailments among these meanings, given in Section 4. We will make crucial use of these entailments in our definition of RECIP, presented informally in Section 7 and formally in Section 9. We leave for future research other issues such as the interaction of RECIP with other semantic operators and the interpretation of reciprocal expressions with quantified antecedents. We also defer discussion of the syntax/semantics interface to another paper.

\section{Why there's a problem about what reciprocals mean}

\subsection{Different sentences have different truth conditions}

Research on the syntax and semantics of reciprocity often centers on sentences such as:

(3) John and Bill saw/kicked/laughed at each other.

Examples in which the reciprocal antecedent denotes a group with two members - here, John and Bill - are particularly easy to analyze. However, attention is often confined to these simple cases. In general, when groups of two members are considered, each group member is required to stand in the stated relation to the other member. ${ }^{1}$

Generalizing to larger antecedent groups, this suggests that

(4) Willow School's fifth-graders know each other.

\footnotetext{
${ }^{1}$ Except for a few cases. Kański (1987) claims that "Their bunk beds are on top of each other" is true when one bunk bed is on top and the other one is under it.
} 
should mean that each Willow fifth-grader knows every other one. In this case, we get the correct truth conditions, as evidenced by the self-contradictoriness of

(5) \# Willow School's fifth-graders know each other, but the oldest one doesn't know the youngest. ${ }^{2}$

However, when reciprocal statements with antecedent groups of larger than two members are considered extensively, these truth conditions turn out to be the wrong ones for many cases. For instance, the following statement from The New York Times can be true despite the impossibility of each group member sitting alongside every other one:

(6) As the preposterous horde crowded around, waiting for the likes of Evans and Mike Greenwell, five Boston pitchers sat alongside each other: Larry Andersen, Jeff Reardon, Jeff Gray, Dennis Lamp and Tom Bolton.

In contrast to (5), note the consistency of

(7) Five Boston pitchers sat alongside each other, Larry Andersen and Tom Bolton sat on the ends, separated by Jeff Reardon, Jeff Gray and Dennis Lamp.

Such examples show that the reciprocal does not always place the same semantic requirements on the interpretation of the sentences containing it. Another example is:

(8) Mrs. Smith's third-grade students gave each other measles.

Clearly, it is impossible for each member of the third-grade class to give measles to every other member. Since measles is a disease that can't be contracted twice, no one can give measles back to whoever gave it to them. Nevertheless, sentence (8) could perfectly well be true.

In addition to these clearcut examples of the reciprocal not imposing the strong requirements that it does in sentences (3) and (4), the literature contains other examples intended to show that the strong interpretation is not the reciprocal's only meaning. Some of these examples are considerably less clearcut than sentences (7) and (8), however, because the reciprocal can be vague. Its vagueness sometimes hampers attempts to provide an accurate characterization of what it means.

\footnotetext{
${ }^{2}$ Further, note the felicity of the following example:

(i) Willow School's fifth-graders know each other, except the oldest one doesn't know the youngest one.

In general, the exception construction is felicitous only in the presence of universal or negative universal quantification over appropriate $n$-tuples. (See Moltmann 1993, although she suggests that universal quantification over pairs may be only implied, not asserted, by reciprocal sentences like (4).) Witness the following contrast:

(ii) Every fifth-grader knows every other fifth-grader, except the oldest one doesn't know the youngest.

(iii) \# Every fifth-grader knows some other fifth-grader, except the oldest one doesn't know the youngest.
} 


\subsection{Truth conditions may be vague or imprecise}

The problems posed by vagueness can be illustrated from many perspectives. For one, consider a bar-room brawl. The statement

(9) The men were hitting each other

may be a perfectly acceptable description of this situation even if some men in the bar do not hit some other ones, as most likely would be the case. Does this constitute a counterexample to the claim that (9) expresses the same sort of truth conditions as (4)? Maybe, but maybe not; it doesn't seem to be a crisp counterexample like (6). The reason is that even the strongest interpretation of the reciprocal may be vague to a certain extent.

Notice that the degree of vagueness decreases when the antecedent denotes a relatively small group, as in

(10) The four men were hitting each other.

This sentence clearly claims that each pair is involved. Similarly, the reciprocal is less vague when its antecedent group is referred to by listing the members.

(11) John, Paul, George, Ringo and Stu were hitting each other

These observations are evidence that sentence (9) is in fact vague, raising the very real possibility that sentence (9) is true of bar-room brawls in which near enough to all pairs of men stand in the relation of hitting, and that no sharp boundary divides what's near enough to all from what isn't.

Although vagueness degrades the theory-testing value of some reciprocal examples, there are still plenty of clear cases like (7) and (8), where any vagueness infecting the truth conditions does not obscure clear differences with the truth conditions of certain other reciprocal sentences. An empirically adequate theory of reciprocal meaning therefore will have to assign different truth conditions depending on a number of factors, including context-dependence.

\subsection{Truth conditions may depend on extra-linguistic context}

Sometimes the context of a reciprocal statement determines a subset comprising just some pairs of distinct members of the antecedent group, what Schwarzschild (1992) calls the operative ones, as the only pairs relevant to the truth or falsity of the reciprocal. Schwarzschild provides the following example:

(12) The prisoners on the two sides of the room could see each other.

He points out that this sentence would be false of a situation in which there are two groups of prisoners, each on one side of a room, and there is an opaque barrier between them; the fact that the prisoners on side A can see other prisoners on side A (and similarly for side B) is not sufficient to make the example true. The operative pairs in this context couple prisoners of side A with ones on side B.

A different sort of context-dependency is that the quantity of operative pairs which the reciprocal says stand in the relation may be determined by context. For example, 
(13) The inspector found peach fruit flies at four different locations within a mile of each other.

would mean that each of the locations is within a mile of every other location, in a context where it was clear that the inspector was systematically criss-crossing the area he was searching. But in a context where it was clear that the inspector was making a linear sweep through a large area to collect a sample of fruit fly traps, the statement would mean that no two consecutive finds of fruit flies were more than a mile apart. Roberts (1987) presents an analysis of reciprocals which reflects the contextual determination of quantificational force; we briefly discuss it below.

Summing up, there are three reasons why what reciprocals mean is problematic:

- The reciprocal (like some type (1,1) quantifiers such as many, few and most) exhibits a certain amount of vagueness.

- Nevertheless, there are genuine shifts in the truth conditions of reciprocal sentences.

- What features of context trigger these shifts and according to what general principles has not been systematically investigated.

Our aim will be to provide an analysis of these shifts, reliably predicting the interpretation of a sentence containing a reciprocal in a given context. We turn now to a discussion of previous proposals for the semantics of reciprocity.

\section{Previously proposed definitions of reciprocity}

Langendoen (1978) was the first to provide an extensive taxonomy of reciprocal meanings. In his pioneering article, he discussed and compared six relations as candidates for the meaning of the reciprocal. We are aware of two other proposals for reciprocal meanings which Langendoen did not discuss; these two come from the work of Kański (1987). Of the eight proposals of Langendoen and Kański, which we enumerate formally and discuss in the subsections of this section, we think that three are genuine meanings of the reciprocal, but the other five are not really things that the reciprocal can mean.

\subsection{Attested definitions}

The examples given as evidence for these reciprocal meanings are false if the definition is not satisfied.

Strong Reciprocity (Langendoen 1978, also called each-the other by Fiengo and Lasnik 1973) is defined as:

(SR) $|A| \geq 2$ and $\quad \forall x, y \in A(x \neq y \rightarrow R x y)$

Informally, SR says that every member of $A$ is related directly by $R$ to every other member.

Examples (4) and (5) attest that the English reciprocal each other can mean Strong Reciprocity.

(14) (=4) Willow School's fifth-graders know each other 
is false unless each member of the set $A$ of Willow fifth-graders stands in the relation $R$ of knowing to every other member of $A$; and (14) is true in that case.

Intermediate Reciprocity (Langendoen 1978) is defined as:

(IR) $|A| \geq 2$ and

$\forall x, y \in A(x \neq y \rightarrow$

for some sequence $z_{0}, \ldots, z_{m} \in A\left(x=z_{0} \wedge R z_{0} z_{1} \wedge \cdots \wedge R z_{m-1} z_{m} \wedge z_{m}=y\right)$

Informally, IR says that every member of $A$ is related directly or indirectly to every other member via the relation $R$. Unlike Strong Reciprocity and most other definitions offered in the literature, Intermediate Reciprocity can hold even when the relation $R$ is asymmetric.

Although Langendoen does not give an example of Intermediate Reciprocity, we believe that (15) is one:

(15) The telephone poles are spaced five hundred feet from each other.

This sentence is true of a situation in which every pole is five hundred feet from the nearest one or ones along the telephone line in either direction. Sentence (15) can easily be true even if not every pole is spaced five hundred feet from every other one (Strong Reciprocity need not hold). But the sentence is false if any two adjacent poles are separated by a distance other than five hundred feet.

The following additional examples of the reciprocal being interpreted as Intermediate Reciprocity are taken from The New York Times.

(16) (=6) As the preposterous horde crowded around, waiting for the likes of Evans and Mike Greenwell, five Boston pitchers sat alongside each other: Larry Andersen, Jeff Reardon, Jeff Gray, Dennis Lamp and Tom Bolton.

(17) "Technically there is enough energy along our coasts to power Britain," he said, "but at this point to deliver 1,000 megawatts to the consumer, you need a wave power plant with machines spread out along 30 miles at some distance from each other, much like underwater fenceposts."

Inclusive Alternative Ordering (Kański 1987) is defined as:

(IAO)

$$
\forall x \in A \exists y \in A(x \neq y \wedge(R x y \vee R y x))
$$

Informally, IAO says that every member $x$ of the set $A$ participates with some other member in the relation $R$ as the first or as the second argument, but not necessarily in both roles. Inclusive Alternative Ordering is the weakest relation that has been proposed as a meaning for the reciprocal. Kański presents the following sentence as an example of Inclusive Alternative Ordering:

(18) The monks lash one another.

He claims that this sentence is true in a situation in which there are ten monks and the lashers are dis joint from the lashees.

A possibly more convincing example is

(19) (= 8) Mrs. Smith's third-grade students gave each other measles. 
This sentence would be true if each member of the class either gave measles to one or more other class members or got measles from another member of the class. It would be false if some class member neither gave measles to any other member of the class nor got the disease from a fellow class member (thus, got it from outside the class if at all). These are exactly the truth conditions of IAO.

\subsection{Unattested definitions}

The purported examples for these definitions are problematic for various reasons.

Partitioned Strong Reciprocity (Langendoen 1978, also called the reciprocal relation by Fiengo and Lasnik 1973 and the distinct subsets relation by Dougherty 1974) is defined as:

(PSR) There is a partition $A_{1}, \ldots, A_{n}$ of $A$ such that for all $i,\left|A_{i}\right| \geq 2$ and

$$
\forall x, y \in A_{i}(x \neq y \rightarrow R x y)
$$

This is simply Strong Reciprocity holding of disjoint subsets that cover the whole set $A$.

Fiengo and Lasnik claim that Partitioned Strong Reciprocity correctly represents the truth conditions of:

(20) The men are hitting each other.

We disagree. We think this sentence means Strong Reciprocity but exhibits a certain amount of vagueness. Fiengo and Lasnik acknowledge (pp. 452-3) "the impossibility of discrete partitioning in an unclear situation" such as a general brawl, and speculate on why reciprocals are somewhat vague. The one piece of evidence they offer for PSR as the correct meaning for example (20) is their intuition that it is false of a situation in which three men are hitting a fourth one, who is hitting them, and no other hitting is going on. We share this intuition, but note it is fully compatible with Strong Reciprocity, since the set of four men is so small that vagueness leaves no latitude for counting this as enough hitting to make the reciprocal true.

In fact, we believe that all examples that appear to illustrate Partitioned Strong Reciprocity instead involve Strong Reciprocity, some of them involving distribution to contextually-given sets as discussed by Schwarzschild (1992). On this view, Partitioned Strong Reciprocity might better be termed Distributed Strong Reciprocity. Consider the following example from The New York Times:

(21) That heart debt is to the small-town society of her youth, which BloodworthThomason describes as a "kind of microcosm of eccentric characters and Southern humor and familial love and extended family and everybody knows everybody and their grandparents knew each other."

This sentence describes a situation in which each citizen of a town satisfies two conditions: that his maternal grandparents knew his paternal grandparents, and vice versa. We can also imagine an example requiring distribution in a different way: 
(22) Many years ago, arranged marriages were common in that town. As for the Smiths, their great-grandparents were complete strangers to each other when they got married. But that all changed 60 years ago; of course, their grandparents knew each other before they got married.

Here, the context requires a different interpretation: Mr. Smith's maternal grandparents knew each other, and so did his paternal grandparents; likewise for Mrs. Smith's grandparents. In this context, example (22) would be judged false if either grandparents of either Smith had an arranged marriage, even if it happened that (for example) the maternal grandmother knew the paternal grandfather, and the paternal grandmother knew the maternal grandfather. It is not sufficient that some partition or other satisfy SR. To make the sentence true, only the contextually salient partition will suffice.

Partitioned Intermediate Reciprocity (Langendoen 1978) is defined as:

(PIR) There is a partition $A_{1}, \ldots, A_{n}$ of $A$ such that for all $i,\left|A_{i}\right| \geq 2$ and

$\forall x, y \in A_{i}(x \neq y \rightarrow$

for some sequence $\left.z_{0}, \ldots, z_{m} \in A_{i}\left(x=z_{0} \wedge R z_{0} z_{1} \wedge \cdots \wedge R z_{m-1} z_{m} \wedge z_{m}=y\right)\right)$

Partitioned Intermediate Reciprocity bears the same relation to Intermediate Reciprocity that Partitioned Strong Reciprocity bears to Strong Reciprocity: Partitioned Intermediate Reciprocity requires Intermediate Reciprocity to hold within disjoint subsets covering $A$.

We have found no example that illustrates Partitioned Intermediate Reciprocity.

Symmetric Reciprocity (Langendoen 1978, also called the unrestricted subsets relation by Dougherty 1974 and Conjunctive Ordering by Kański 1987) is defined as:

(SmR)

$$
\forall x \in A \exists y \in A(x \neq y \wedge R x y \wedge R y x)
$$

Informally, this says that every member of $A$ has a 'partner' with whom the relation $R$ holds in both directions. Dougherty (1974) argues that Symmetric Reciprocity gives the proper truth conditions for example (23).

(23) John, Bill, Tom, Jane, and Mary had relations with each other.

He claims (1974, page 14) that this sentence can be true even if "only heterosexual relations are involved" - if (for example) Jane had relations with both John and Bill, but John and Bill did not have relations with each other. In this situation, there is no way to partition the set $\{\mathrm{John}$, Bill, Tom, Jane, Mary $\}$ into subsets within which each member bears the have relations with relation to every other member.

We agree that the truth conditions for example (23) are as Dougherty describes; however, this and all other apparent examples of Symmetric Reciprocity that we have found involve relations that are inherently symmetric. Sentence (23) may well just attest the possibility of interpreting the reciprocal as Inclusive Alternative Ordering. 
Weak Reciprocity (Langendoen 1978) is defined as:

$$
\forall x \in A \exists y, z \in A(x \neq y \wedge x \neq z \wedge R x y \wedge R z x)
$$

Informally, WR says that every member of the group $A$ participates in the relation $R$ both as the first and as the second argument. Weak Reciprocity is the weakest of the relations discussed by Langendoen, but is stronger than Kański's Inclusive Alternative Ordering.

Langendoen (1978, page 183) offers the following example for Weak Reciprocity (in lieu of Partitioned Intermediate Reciprocity):

(24) They are at least as heavy as one another.

We are instructed to evaluate this sentence in a situation in which they refers to a group "consisting of five individuals, of whom two weigh $50 \mathrm{~kg}$ each, one weighs $60 \mathrm{~kg}$, and two weigh $70 \mathrm{~kg}$ each." Langendoen judges the sentence to be true in this situation, and thus to exhibit the truth conditions that Weak Reciprocity would assign, although he states (and we agree) that the sentence is "bizarre independent of the truth or falsity of the assertion that it makes". Unfortunately, it is not easy to come up with more natural sentences that would readily distinguish Weak Reciprocity from independently attested definitions.

In fact, all examples in the literature we are aware of which have been proferred as attesting Weak Reciprocity have as their scope an inherently symmetric relation. When the scope relation $R$ is symmetric, Inclusive Alternative Ordering implies Weak Reciprocity, Partitioned Intermediate Reciprocity and Symmetric Reciprocity as we shall see. Thus, we believe that these examples are best regarded as exemplifying IAO and not WR, PIR or SmR, since IAO is also exemplified by nonsymmetric cases whose meaning is definitely none of the other definitions.

Exclusive Alternative Ordering (Kański 1987) is defined as:

(EAO) $\quad \forall x \in A \exists y \in A(x \neq y \wedge(R x y \vee R y x) \wedge \neg(R x y \wedge R y x))$

Informally, EAO says that every member of the group $A$ stands asymmetrically in the relation $R$ with some other member. This is a rather peculiar requirement.

Kański claims that Exclusive Alternative Ordering is exemplified by asymmetric relations $R$, such as:

(25) The students followed each other (into the room).

However, we do not believe that the reciprocal ever implies that the relation $R$ does not hold symmetrically. Of course, we agree with Kański that sentences containing reciprocals can be true even in case the relation $R$ is asymmetric, as in the case of example (25). As an account of the truth conditions of such sentences, IAO is equivalent to EAO.

The relationships among these various definitions of reciprocity can be seen more clearly if they are uniformly stated in terms of more basic relations; additionally, this enables the statement of implications among these definitions. 


\section{Logical analysis of all previously proposed readings}

Parameterizing the differences between the various definitions of RECIP identified by Langendoen and others, as suggested by Dag Westerståhl (p.c.), is useful in understanding the logical relations among these definitions. We analyze $\operatorname{RECIP}(A, R)$ as $Q\left(A, R^{\sharp}\right)$. $Q$ is either FUL $\backslash I$ or TOT $\backslash I$ :

$$
\begin{array}{rll}
\operatorname{FUL}(A, R) & \stackrel{\text { df }}{\leftrightarrow} & R \uparrow A=A \times A \\
\operatorname{TOT}(A, R) & \stackrel{\text { df }}{\leftrightarrow} & \operatorname{dom}(R \uparrow A)=A \\
\operatorname{FUL} \backslash I(A, R) & \stackrel{\text { df }}{\leftrightarrow} & \operatorname{FUL}(A, R \cup I) \\
\operatorname{TOT} \backslash I(A, R) & \stackrel{\text { df }}{\leftrightarrow} & \operatorname{TOT}(A, R \backslash I)
\end{array}
$$

$I$ is the identity relation. The definitions of FUL $\backslash I$ and TOT $\backslash I$ capture the fact that truth conditions for the reciprocal never depend on an individual bearing or not bearing the relation $R$ to itself.

$R^{\sharp}$ is either $R\lceil A(=R \cap(A \times A)$ ) or the result of applying to it one or more of three operations, in any order. The three operations that can be used to get $R^{\sharp}$ from $R$ are ${ }^{\wedge}, \vee$, and ${ }^{+}$:

$$
\begin{array}{lll}
R^{\wedge} & \stackrel{\mathrm{df}}{=} & R \cap R^{-1} \\
R^{\vee} & \stackrel{\mathrm{df}}{=} & R \cup R^{-1} \\
R^{+} & \stackrel{\mathrm{df}}{=} & \text { transitive closure of } R
\end{array}
$$

Four of Langendoen's six definitions and one of Kański's two new ones are expressible in these terms. Below, for $R$, read $R\lceil A$ :

$$
\begin{array}{ll}
\text { Strong Reciprocity: } & \text { FUL } \backslash I(A, R) \\
\text { Symmetric Reciprocity: } & \text { TOT } \backslash I\left(A, R^{\wedge}\right) \\
\text { Intermediate Reciprocity: } & \text { FUL } \backslash I\left(A, R^{+}\right) \\
\text {Partitioned Intermediate Reciprocity: } & \text { TOT } \backslash I\left(A,\left(R^{+}\right)^{\wedge}\right) \\
\text { Inclusive Alternative Ordering: } & \text { TOT } \backslash I\left(A, R^{\vee}\right)
\end{array}
$$

Partitioned Strong Reciprocity and Kański's Exclusive Alternative Ordering are not definable in these terms. Weak Reciprocity is not definable in these terms, either, but can be expressed as the following, using conjunction and the relational converse:

$$
\text { Weak Reciprocity: } \quad \text { TOT } \backslash I(A, R) \wedge \text { TOT } \backslash I\left(A, R^{-1}\right)
$$

The three operations ${ }^{\wedge}, \vee$, and ${ }^{+}$generate eight different relations from $R$, and they stand in the inclusion relation in the following way ( $\rightarrow$ means $\subseteq$ ):

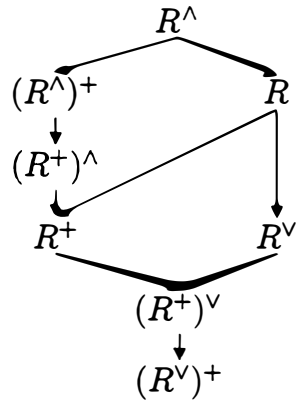


The given format of defining RECIP gives rise to 10 non-equivalent possible definitions, of which five are new possibilities that have not been considered in the literature. The following graph depicts the implications among these definitions, and includes Partitioned Strong Reciprocity, which is not definable in these terms, as well as Weak Reciprocity:

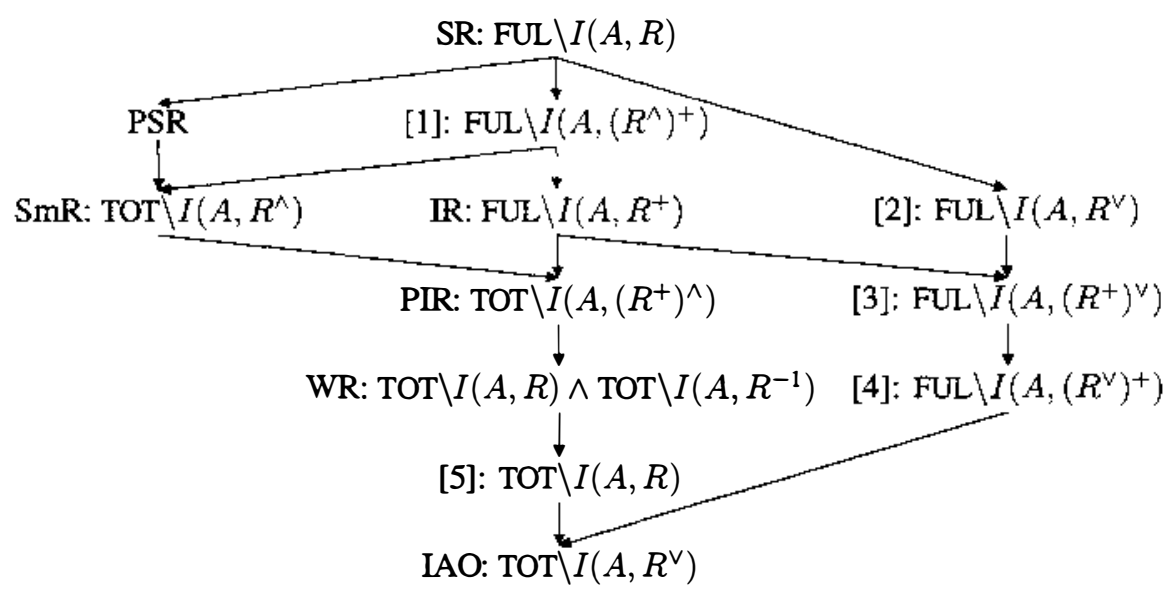

The RECIP relations labeled [1]-[5] are possible candidates for the meaning of RECIP that have not been previously discussed in the literature.

\section{Attested new definitions of reciprocity}

It appears that some of these new definitions are attested by actual uses of reciprocals. In J.M. Barrie's "Peter Pan," for instance, the sentence

(26) "The captain!" said the pirates, staring at each other in surprise.

states that each pirate stared at another one, although perhaps not every pirate was stared at by another one. These truth conditions are exactly those of the new definition [5].

In The New York Times, we find the following description of the National Cathedral in Washington, DC:

(27) Instead, countless stones - each weighing an average of 300 pounds - are arranged on top of each other and are held in place by their own mass and the force of flying buttresses against the walls.

Definition [4] would make this sentence an accurate description of the cathedral, which is built of stones arranged in staggered, overlapping patterns like a brick wall. If [4] is what (27) means, the sentence asserts that the stones form a single connected structure; it would be false if, for example, the stones were arranged in a multiplicity of piles, each pile separate from the others. More evidence is needed before a secure conclusion can be reached, but we believe at least some of these systematically-generated new definitions for the reciprocal's meaning are also attested. 
Because there apparently are reciprocals sentences whose meaning is a previously unattested definition - most particularly IR, but also [4] and [5] - we provisionally assume that other candidates among the ones we have canvassed may turn out also to be possible meanings of reciprocals. We do henceforth dismiss PSR and EAO, however, as not merely unattested but unallowed meanings for the reciprocal. $^{3}$ The problematic status of the evidence for some reciprocal meanings, discussed in Section 3, comes in some instances from the fact that the nature of the scope relation $R$ can render some of these meanings indistinguishable.

\section{Dependence of partial ordering on special properties of scope}

Under certain circumstances, some of the definitions discussed in the previous section become equivalent; some of these cases were alluded to in Section 3, in the discussion of previously proposed but unattested reciprocal meanings. In case the scope relation $R$ is transitive and symmetric, for example, the eleven possible reciprocal meanings collapse to only two separate cases. Care must be taken to control for these factors in considering evidence for the potential reciprocal meanings under discussion.

Symmetric $R$ If $R$ is symmetric, $R=R^{-1}=R^{\wedge}=R^{\vee}$ and the partially ordered possibilities reduce to:

$$
\begin{gathered}
\text { SR, [2] } \\
\text { IR, [1], [3], [4] } \\
\text { IAO, SmR, PIR, WR, [5] }
\end{gathered}
$$

Transitive $R$ If $R$ is transitive, $R=R^{+}$and the partially ordered possibilities reduce to:

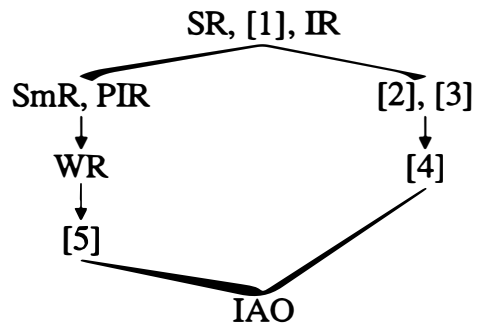

Transitive and symmetric $R$ If $R$ is transitive and symmetric, the partially ordered possibilities reduce to:

$$
\begin{aligned}
& \mathrm{SR}, \mathrm{IR},[1],[2],[3],[4] \\
& \text { IAO, SmR, PIR, WR, [5] }
\end{aligned}
$$

Asymmetric $R$ If $R$ is asymmetric, $R^{\wedge}=\emptyset$ and SR, [1], and SmR are necessarily false (assuming $|A| \geq 2$ ).

\footnotetext{
${ }^{3}$ We emphatically reaffirm, however, that reciprocal VPs can be distributed over the denotation of their subject NPs, as other VPs can. This is how examples (21) and (22) are interpreted.
} 


\section{The Strongest Meaning Hypothesis}

We spoke informally in preceding sections of the reciprocal's meaning, reading, interpretation or truth conditions varying with context. To be slightly more precise, we were saying that because of what it means, the reciprocal can give different truth conditions to different statements. It is time to get more precise still. We have come to the main point of this paper: systematic investigation of the factors underlying the shifts in reciprocals' truth conditions which were noted in the literature we cite.

On one important issue - whether the reciprocal itself is ambiguous let us disclose our conclusion in advance. Do we claim each other is ambiguous, somewhat as the adjective light means bright (the opposite of dark) and also means low in weight (the opposite of heavy) according to a speaker's choice? Or do we claim each other has a single meaning that exploits an utterance's context in determining the utterance's truth conditions, somewhat as here unambiguously means this place, and refers on each occasion to wherever it is uttered?

We believe the latter: the reciprocal has just one meaning, a flexible or context-sensitive one in virtue of which it makes varying contributions to a statement's truth conditions depending on the context in which it appears. The variation is not just in 'fudge factor' or in quantificational force; the reciprocal's meaning is actually capable in appropriate contexts of giving rise to many of the forms of truth conditions discussed in the previous sections. What, precisely, is that contextsensitive meaning then? discovered.

The answer we propose is based on an empirical generalization we have

(SMH) The Strongest Meaning Hypothesis:

A reciprocal sentence is interpreted as expressing the logically strongest candidate truth conditions which are not contradicted by known properties of the relation expressed by the reciprocal scope when restricted to the group argument.

We now examine the evidence supporting this generalization, using the examples discussed earlier and the truth conditions we determined that they have.

In a large collection of the examples we have discussed, the reciprocal means Strong Reciprocity. The Strongest Meaning Hypothesis is simple to verify in these cases - just check for each reciprocal sentence interpreted as a Strong Reciprocal that there are circumstances under which it would be true, given that the reciprocal is interpreted that way. As one example,

(31) (=4) Willow School's fifth-graders know each other.

is satisfiable when interpreted as expressing SR, given the properties of knowing. Thus the SMH predicts that the truth conditions of sentence (31) are SR, since this is the logically strongest candidate for truth conditions of the reciprocal.

An example of a different sort, meaning not SR but Intermediate Reciprocity, is

(32) Five Boston pitchers sat alongside each other.

modeled on sentence (6) from The New York Times. Here the reciprocal's scope is 
the relation of sitting alongside. Note first that sitting alongside is a symmetric relation. As pointed out in Section 6, this renders many of the twelve truth conditions discussed earlier equivalent to other ones, collapsing the lattice to only three possibilities: SR(=[2]), the strongest; IR(=[1],[3],[4]); and IAO(=SmR,PIR,[5],WR), the weakest. Another important property of sitting alongside is that its restriction to any group of pitchers is 'two-valued': a pitcher can sit alongside no more than two other pitchers, since pitchers are people and thus have only two sides. When the group $A$ consists of more than three members, then, SR cannot hold. Of course, IAO is consistent with the properties of sitting alongside; but so is the logically stronger IR, so the SMH predicts that IR is the meaning.

The scope of

(33) (= 8) Mrs. Smith's third-grade students gave each other measles.

is the relation of giving measles to. Note that giving measles is necessarily asymmetric, so that SR, SmR, and [1] cannot hold. In fact, even [5] cannot hold, since interpretation [5] of (33) makes it entail

(34) Each third grader gave another third grader measles.

A person can only have measles once; however, if there are finitely many third graders, (34) entails the existence of a collection of third graders who passed measles around in a circle, which is impossible without one or more of them getting measles twice. So interpretation [5] of (33), which entails (34), is necessarily false. This leaves Inclusive Alternative Ordering as the interpretation that the SMH predicts for (33) if interpretation [4] is necessarily false.

However [4] merely entails that

(35) any two third graders are connected by a chain of third graders each of whom either gave measles to or got measles from the next one.

Together with the impossibility of getting measles twice, this entails that there is one third grader from whom all the others got measles either directly or indirectly. This disease transmission pattern is actually possible; so the Strongest Meaning Hypothesis actually predicts that (33) does not mean IAO but something stronger such as [4].

But we suggested in Subsection 3.1 that (33) means IAO. Can we reconcile the Strongest Meaning Hypothesis with the observations considered there? The answer is yes if we accept that there is some vagueness in the meaning of (33). While the Strongest Meaning Hypothesis predicts this sentence means one third grader was the only point of entry of measles into the class, we could allow that there might be a few more points of entry, but still only a small number, if the class is large enough. This is analogous to the Strongest Meaning Hypothesis predicting that sentence (9) means Strong Reciprocity, but vagueness allowing some men not to hit some other ones when the sentence is true of a bar-room brawl.

Note a further problem for the $\mathrm{SMH}$, which cannot be solved in the same way. Interpretation [3] of sentence (33) is also consistent. It entails that measles were propagated through the third-grade class in a linear, nonbranching chain. Clearly the sentence does not mean this. This is a serious problem if [3] is truly a candidate 
meaning for the reciprocal. So far, however, we have not found a convincing example that attests [3]. We conclude tentatively that [3] is not a candidate interpretation of the reciprocal at all. ${ }^{4}$

The SMH correctly predicts that sentence

(36) Their bunk beds are on top of one another.

discussed by Kański (1987), means IAO rather than SR even though the group consists of only two members. This successful prediction of the SMH is unfortunately accompanied by an incorrect one, however, namely that any reciprocal sentence will mean IAO if all stronger candidates are unsatisfiable. For instance, the SMH predicts that

(37) \# Those two people are each other's mother.

is true just in case one of the people is the mother of the other. In fact, contrary to the $\mathrm{SMH}$, this sentence means Strong Reciprocity and is necessarily false. These examples show the need to revise the SMH in a way that preserves the prediction that the reciprocal means the strongest possible candidate but drops the insistence that only satisfiable candidates are possible.

\section{Alternative proposals for reciprocal meaning: Why they fail}

Varying quantification over individuals? Other researchers have proposed criteria for determining reciprocal meanings which appeal to the context in which the reciprocal appears. Roberts (1987) proposes that reciprocals have a single meaning, but one that is context dependent, so that the reciprocal can make different semantic contributions depending on the context in which it appears. Following a suggestion of Emmon Bach's, she proposes to use the context-sensitive quantifier ENOUGH to define reciprocal meaning (Roberts, 1987, page 142):

$$
\text { ENOUGH }_{1} x \in A . \mathrm{ENOUGH}_{2} y, z \in A . x \neq y \wedge x \neq z \wedge R(x, y) \wedge R(z, x)
$$

Roberts notes that varying the interpretation of ENOUGH yields Strong Reciprocity and Weak Reciprocity as subcases: Interpreting both instances of ENOUGH as universal quantification gives Strong Reciprocity, while interpreting the first instance of ENOUGH as universal quantification and the second as existential quantification gives Weak Reciprocity. In fact, Roberts' formula (38) does not quite achieve the intended effect. To get Strong Reciprocity by interpreting ENOUGH $\mathrm{EN}_{1}$ and $\mathrm{ENOUGH}_{2}$ as universal quantifiers, the conditions $x \neq y$ and $x \neq z$ must appear in the restriction of the second universal quantifier. This problem can easily be repaired by modifying (38) as follows:

(39) $\mathrm{ENOUGH}_{1} x(x \in A$,

$$
\left.\mathrm{ENOUGH}_{2} y, z(y \in A \wedge z \in A \wedge x \neq y \wedge x \neq z, R(x, y) \wedge R(z, x))\right)
$$

Here, $\mathrm{ENOUGH}_{1}$ is a type $(1,1)$ quantifier, and $\mathrm{ENOUGH}_{2}$ is a type $(2,2)$ quantifier.

We agree with Roberts that it is necessary to appeal to contextual factors to determine the semantic contribution of the reciprocal; however, the particular

\footnotetext{
${ }^{4}$ Note that PIR, which, like [3], involves transitive closure $\left({ }^{+}\right)$inside another operator, is also unattested. It may be that the use of transitive closure in our parameterization scheme should be reconsidered.
} 
appeal to context that she makes is insufficient to capture the variety of reciprocal meanings that are found. For instance, there is no way to vary the interpretation of ENOUGH to produce the truth conditions required for Intermediate Reciprocity, though, as we have seen, some reciprocal sentences impose these requirements:

(40) The freeway exits are spaced five miles from each other.

Intuitively, the difficulty with Roberts's proposal for reciprocal meanings is that it assumes that varying the force of quantification over individuals is sufficient to distinguish different reciprocal meanings. Examples such as (40) show that to properly distinguish different reciprocal meanings, it is necessary to quantify over pairs of individuals standing in the relation $R$, which comes from the polyadic quantifier RECIP. ${ }^{5}$

Unambiguous reciprocal meaning? Langendoen (1978), in proposing various possible reciprocal meanings, shared a common assumption with a number of other researchers studying the semantics of reciprocity: that the reciprocal makes a uniform semantic contribution on every occasion of its use. Langendoen's intention, then, was to discover the core of truth conditions included in every instance of use of the reciprocal; this common meaning must, he reasoned, constitute the semantic contribution of the reciprocal. Thus, if he could find data counterexemplifying a proposed reciprocal meaning, then that meaning could be ruled out. His analysis, then, rules out all but the weakest meaning he discusses, Weak Reciprocity, as the correct meaning of the reciprocal.

Of course, Langendoen wouldn't deny that examples like (41) appear to express Strong Reciprocity.

(41) (=4) Willow School's fifth-graders know each other.

An advocate of his position needs to explain why such examples appear to mean something stronger than Weak Reciprocity. Doing so will presumably involve appeal to pragmatic strengthening of the proposition that according to them is the sentence's literal meaning.

One way such strengthening might occur is through conversational implicature. A hallmark of conversational implicatures is their cancelability. The added

\footnotetext{
${ }^{5}$ Under reasonable assumptions, one can prove that no choice of $\mathrm{ENOUGH}_{1}$ and $\mathrm{ENOUGH}_{2}$ makes (39) capture the truth conditions of Intermediate Reciprocity. Since the variables $y$ and $z$ in (39) vary independently, (39) must be equivalent to the following formula on models where $R$ is symmetric: $\mathrm{ENOUGH}_{1} x\left(x \in A, \mathrm{ENOUGH}_{3} y(y \in A \wedge x \neq y, R(x, y))\right)$,

Here, $\mathrm{ENOUGH}_{1}$ is as before, and $\mathrm{ENOUGH}_{3}$ is a suitable quantifier of type $(1,1)$. Nowitis easy to prove that Intermediate Reciprocity (IR) cannot be equivalent to (i) on all models where $R$ is symmetric, provided that $\mathrm{ENOUGH}_{1}$ and $\mathrm{ENOUGH}_{3}$ are permutation invariant, as all 'logical' quantifiers are (see van Benthem 1986 or Westerståhl 1989). It suffices to observe that while IR distinguishes the following two models, (i) cannot:

(ii)

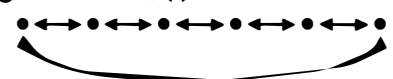

(Here, all individuals are in $A$ and arrows indicate that $R$ holds in both directions.) Since (39), but not $\mathbb{R}$, is equivalent to (i) on all symmetric models, (39) and $\mathbb{R}$ cannot be equivalent.
} 
strength of (41) over Weak Reciprocity does not, however, seem to be cancelable, as evidenced by the inconsistency of (42):

(42) (= 5) \# Willow School's fifth-graders know each other, but the oldest one doesn't know the youngest.

Thus it does not seem that a Gricean approach to strengthening can do justice to the facts. Moreover, conversational implicatures are highly ad hoc, their specific content varying enormously with changes in the situation of utterance. However, the literature on reciprocals suggests that only a few different forms of proposition are possible strengthened interpretations of a reciprocal sentence. It is completely unclear, at best, how strengthening Weak Reciprocity by conversational implicature could function so as always to yield one of this small number of stronger propositions as what is implicated.

\section{Formalizing the Strongest Meaning Hypothesis}

The Strongest Meaning Hypothesis is that a reciprocal sentence means the logically strongest one of these candidates that is satisfiable. To make this precise, we need a set $\Theta_{R}$ of axioms giving the known properties of each relation $R$ that could be the scope of a reciprocal statement, such as symmetry or transitivity. Suppose we have an 'oracle' $\Theta$ that gives us such a set of axioms for each relation.

Recall the ten candidate truth conditions we still have under consideration for reciprocals - six previously proposed candidates, supplemented with four additional ones generated by Westerståhl's suggestion for regularizing these. Keep in mind the implication lattice of these ten candidate definitions.

Then we define $\operatorname{RECIP}(A, R)$ as follows, where $p$ and $q$ range over these ten definitions of RECIP:

$$
\bigvee_{q}\left(q \wedge \bigwedge_{p}\left(\left(\Theta_{R} \models(p \rightarrow q) \wedge \Theta_{R} \not \models(q \rightarrow p)\right) \rightarrow \Theta_{R} \models \neg p\right)\right)
$$

Given a particular oracle $\Theta$, RECIP means the disjunction of all the strongest candidate meanings $q$ such that $q$ holds, and any strictly stronger meaning $p$ is inconsistent with the axioms $\Theta_{R}$. Note that:

$$
\begin{aligned}
& \operatorname{RECIP}(A, R) \leftrightarrow \quad \bigvee \quad r \\
& r \text { and no stronger candidate is consistent with } \Theta_{R}
\end{aligned}
$$

When there is a unique strongest candidate definition which is consistent with the logical properties of $R$, the axioms for $R$ imply that $\operatorname{RECIP}(A, R)$ is equivalent to that candidate definition. When there are several such candidates, the axioms for $R$ imply that $\operatorname{RECIP}(A, R)$ is equivalent to the disjunction of those candidates.

\section{Conclusion}

We have tried to make sense of the plethora of apparent meanings that RECIP can have, taking into account the apparent lack of ambiguity of RECIP, and to give the best possible systematization of previous proposals for reciprocal meaning: the strongest meaning that is consistent with known facts is the one that will apply. 


\section{Acknowledgments}

This work was supported in part by National Science Foundation grant number SBR93-19371, and also supported by the Information Technology Promotion Agency, Japan, as part of the R\&D of Basic Technology for Future Industries "New Models for Software Architecture" sponsored by NEDO (New Energy and Industrial Technology Development Organization). For invaluable assistance, we would like to thank Yasunari Harada, Yookyung Kim, Cleo Condoravdi, Mark Gawron, Chris Piñón, Alex Alsina, and Stuart Shieber.

\section{References}

Dougherty, Ray C. 1974. The syntax and semantics of each other constructions. Foundations of Language, 12:1-47.

Fiengo, Robert and Howard Lasnik. 1973. The logical structure of reciprocal sentences in English. Foundations of Language, 9(4):447-468.

Higginbotham, James. 1980. Reciprocal interpretation. Journal of Linguistic Research, 1(3):97-117.

Kański, Zbigniew. 1987. Logical symmetry and natural language reciprocals. In Proceedings of the 1987 Debrecen Symposium on Language and Logic, pages 49-68, Budapest. Akadémiai Kiadó.

Langendoen, D. Terence. 1978. The logic of reciprocity. Linguistic Inquiry, 9(2):177-197.

Lønning, Jan Tore. 1989. Some Aspects of the Logic of Plural Noun Phrases. Ph.D. thesis, University of Oslo.

Moltmann, Friederike. 1992. Reciprocals and same/different: Towards a semantic analysis. Linguistics and Philosophy, 15(4):411-462.

Moltmann, Friederike. 1993. Exception sentences and polyadic quantification. MS, UCLA.

Roberts, Craige. 1987. Modal Subordination, Anaphora, and Distributivity. Ph.D. thesis, University of Massachusetts at Amherst.

Schwarzschild, Roger. 1992. Types of plural individuals. Linguistics and Philosophy, 15(6):641-675.

van Benthem, Johan. 1986. Essays in logical semantics. D. Reidel, Dordrecht.

van Benthem, Johan. 1989. Polyadic quantifiers. Linguistics and Philosophy, 12(4):437-464.

Westerståhl, Dag. 1989. Quantifiers in formal and natural languages. In D. Gabbay and F. Guenthner, editors, Handbook of philosophical logic, volume IV. D. Reidel, Dordrecht, pages 1-131.

Williams, Edwin. 1991. Reciprocal scope. Linguistic Inquiry, 22(1):159-173. 